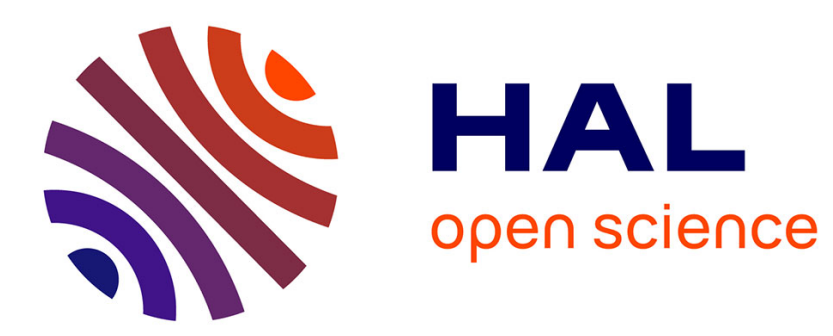

\title{
Étude d'un effet photothermique particulier dans les semi-conducteurs
}

\author{
J. Oualid
}

\section{To cite this version:}

J. Oualid. Étude d'un effet photothermique particulier dans les semi-conducteurs. Revue de Physique Appliquée, 1967, 2 (2), pp.72-78. 10.1051/rphysap:019670020207200 . jpa-00242779

\section{HAL Id: jpa-00242779 https://hal.science/jpa-00242779}

Submitted on 1 Jan 1967

HAL is a multi-disciplinary open access archive for the deposit and dissemination of scientific research documents, whether they are published or not. The documents may come from teaching and research institutions in France or abroad, or from public or private research centers.
L'archive ouverte pluridisciplinaire HAL, est destinée au dépôt et à la diffusion de documents scientifiques de niveau recherche, publiés ou non, émanant des établissements d'enseignement et de recherche français ou étrangers, des laboratoires publics ou privés. 


\title{
ÉTUDE D'UN EFFET PHOTOTHERMIQUE PARTIGULIER DANS LES SEMI-GONDUGTEURS
}

\author{
Par J. OUALID, \\ Laboratoire d'Électricité et d'Héliotechnique, Laboratoire de Physique SPCN III, \\ Faculté des Sciences, Marseille-Saint-Jérôme.
}

\begin{abstract}
Résumé. - On présente l'effet photothermique dans les semi-conducteurs qui consiste en une augmentation apparente de la conductibilité thermique d'un semi-conducteur éclairé par un rayonnement d'énergie suffisante. On établit théoriquement la distribution de température dans une plaquette semi-conductrice éclairée sur une des faces par une impulsion lumineuse brève. On calcule une expression approchée de la variation relative de la conductibilité thermique qui permet de déterminer directement le rendement quantique de création de paires électron-trou et leur longueur de diffusion. On décrit enfin une expérience sur un échantillon de germanium qui a permis de vérifier assez correctement les résultats théoriques, ce qui justifie les hypothèses formulées.
\end{abstract}

Abstract. - When a semi-conductor is illuminated with a strong enough radiation pulse, an increase of thermal conductivity is observed. This so called photothermal effect is studied in the case of a semi-conductor slab illuminate on one face by a short pulse of light. A theoretical temperature distribution and an approximated expression for the relative variation of the thermal conductivity, leading to quantum efficiency for electron-hole creation and scattering length measurements, are given. An experiment with a germanium sample is described. The theoretical results and the formulated hypotheses are fairly well verified.

I. Définition de l'effet photothermique. - Lorsqu'on éclaire la surface d'un échantillon semiconducteur (S.C.) par un rayonnement d'énergie suffisante, il y a, au voisinage de la surface, création de paires électron-trou qui diffusent vers l'intérieur de l'échantillon. Chaque paire transporte une énergie voisine de la largeur de bande interdite $E_{\mathrm{G}}$. Les paires électron-trou cèdent en se recombinant leur énergie au réseau. La distribution de température de l'échantillon sera modifiée et par suite on observera expérimentalement une variation apparente de la conductibilité thermique que l'on appelle effet photothermique.

Get effet a été mis en évidence par Gartner [1], dans le cas du germanium. Timberlake [2] et Weill [3] l'ont signalé au cours de mesures de la conductibilité thermique de différents semi-conducteurs. Il n'a pas encore fait l'objet d'une étude systématique, du fait des difficultés expérimentales rencontrées et aussi de la complexité avec laquelle cet effet est décrit analytiquement.

Nous avons tenté d'établir une théorie simplifiée de cet effet dans le cas particulier où le S.C. est éclairé sur une des faces par une impulsion lumineuse très brève et nous avons essayé de vérifier expérimentalement les résultats de notre étude théorique.
II. Théorie de l'effet photothermique dans le cas d'un semi-conducteur éclairé par une impulsion lumineuse. - Pour simplifier la description de l'effet photothermique, nous avons été amenés à faire un certain nombre d'hypothèses.

$1^{\circ} \mathrm{La}$ surface du S.C. de type $n$ est éclairée par une impulsion lumineuse monochromatique de durée $\theta$ suffisamment brève pour l'assimiler à une fonction $\delta$.

$2^{\circ}$ La lumière est totalement absorbée à la surface de l'échantillon et il n'y a pas de création en volume de paires électron-trou.

$3^{\circ}$ Les paires électron-trou créées à la surface par des photons d'énergie $h v$ supérieure à la largeur de bande interdite $E_{\mathrm{G}}$ se thermalisent instantanément. L'énergie dissipée est absorbée par le réseau à la surface éclairée. Par suite, chaque paire transportera par diffusion une énergie voisine de $E_{\mathrm{G}}$.

$4^{\circ}$ La surface de l'échantillon est parfaitement polie afin de négliger la recombinaison superficielle des paires électron-trou.

$5^{\circ}$ L'énergie $E_{\mathrm{G}}$ provenant de la recombinaison d'une paire électron-trou est intégralement retransmise au réseau cristallin.

$6^{\circ}$ La durée de vie $\tau$ des paires électron-trou est constante ainsi que la longueur de diffusion $L$. 
$7^{0}$ Les surfaces latérales de l'échantillon sont parfaitement isolées thermiquement.

$8^{\circ}$ Le problème est à une dimension et la plaquette semi-conductrice semi-infinie.

On peut alors écrire les équations de conservation de l'énergie et de la charge sous la forme :

$$
\begin{aligned}
& \frac{\partial(\Delta p)}{\partial t}-D \frac{\partial^{2}(\Delta p)}{\partial x^{2}}+\frac{\Delta p}{\tau}=0 \\
& \frac{\partial T}{\partial t}-D_{\mathrm{T}}^{(0)} \frac{\partial^{2} T}{\partial x^{2}}-\frac{E_{\mathrm{G}}}{\rho c \tau} \Delta p=0
\end{aligned}
$$

où $\Delta p$ est la densité des paires électròn-trou, $D$ le coefficient de diffusion ambipolaire, $D_{\mathrm{T}}^{(0)}$ le coefficient de diffusion thermique pour le S.C. non éclairé défini $\operatorname{par} D_{\mathrm{T}}^{(0)}=\frac{K^{(0)}}{\rho c}$ où $K^{(0)}$ est la conductibilité thermique, $\rho$ la masse volumique et $c$ la chaleur massique.

Ces deux équations associées aux conditions initiales et aux limites suivantes :

$$
\begin{aligned}
& -D\left(\frac{\partial(\Delta p)}{\partial x}\right)_{x=0}=\frac{\eta P \theta \delta(t)}{h \nu} ; \\
& \Delta p(\infty, t)=0 ; \Delta p(x, 0)=0 \\
& -K^{(0)}\left(\frac{\partial T}{\partial x}\right)_{x=0}=P \theta\left(\begin{array}{c}
\left.1-\eta \frac{E_{\mathrm{G}}}{h \nu}\right) \delta(t) ; \\
T(\infty, t) ; T(x, 0)=0
\end{array}\right.
\end{aligned}
$$

donnent la distribution de température dans le barreau semi-conducteur.

$P$ est la puissance lumineuse absorbée par unité de surface, $\eta$ est le rendement quantique de création des paires électron-trou.

Soient $\Delta \bar{p}$ et $\bar{\varphi}$ les transformées de Laplace de $\Delta p$ et de $\varphi=\frac{\partial T}{\partial x}$. Les équations (1) et (2) s'écrivent
alors :

$$
\frac{\partial^{2}(\Delta \bar{p})}{\partial x^{2}}=q_{\mathrm{p}}^{2} \Delta \bar{p}
$$

avec $\quad q_{\mathrm{p}}^{2}=\frac{p}{D}+\frac{1}{L^{2}} \quad$ et $\quad L^{2}=D \tau$

$-D\left(\frac{\partial(\Delta \bar{p})}{\partial x}\right)_{x=0}=\frac{\eta P \theta}{h \nu} ; \Delta \bar{p}(\infty, p)=0 ;$
$\Delta \bar{p}(x, 0)=0$.
L'équation (5) s'intègre immédiatement et on a :

$$
\Delta \bar{p}=\frac{\eta P \theta}{h \nu D} \frac{\exp \left(-q_{\mathrm{p}} x\right)}{q_{\mathrm{p}}}\left({ }^{1}\right) .
$$

La transformée de Laplace de l'équation (2) s'écrit alors :

$$
\frac{\partial^{2} \bar{\varphi}}{\partial x^{2}}=q_{\mathrm{T}}^{2} \bar{\varphi}+\frac{E_{\mathrm{G}} \eta P \theta}{\rho c \tau h \nu D} \exp \left(-q_{\mathrm{p}} x\right)
$$$$
\text { avec } \quad q_{\mathrm{T}}^{2}=\frac{p}{D_{\mathrm{T}}^{(0)}} \text {. }
$$

Associée aux conditions aux limites :

$$
\begin{aligned}
\bar{\varphi}(0, p)=-\frac{P\left(1-\eta E_{\mathrm{G}} / h \nu\right) \theta}{K^{(0)}} ; \\
\bar{\varphi}(\infty, p)=0 ; \quad \bar{\varphi}(x, 0)=0 .
\end{aligned}
$$

L'équation (8) s'intègre immédiatement et donne :

$$
\begin{aligned}
& \bar{\varphi}=-\frac{P \theta}{K^{(0)}}\left(1-\eta \frac{E_{\mathrm{G}}}{h \nu}\right) \exp \left(-q_{\mathrm{T}} x\right) \\
& -\frac{\eta E_{\mathrm{G}}}{h \nu} \cdot \frac{P \theta}{\rho c L^{2} D_{\mathrm{T}}^{(0)}} \cdot \frac{\exp \left(-q_{\mathrm{T}} x\right)-\exp \left(-q_{\mathrm{p}} x\right)}{q_{\mathrm{p}}^{2}-q_{\mathrm{T}}^{2}} .
\end{aligned}
$$

En tenant compte du fait que $D \gg D_{\mathrm{T}}^{(0)}$ (pour le germanium $D \# 50 \mathrm{~cm}^{2} / \mathrm{s}, D_{\mathrm{T}}^{(\omega)} \# 0,4 \mathrm{~cm}^{2} / \mathrm{s}$ ), on peut écrire $\bar{\varphi}$ sous la forme :

$$
\begin{gathered}
\bar{\varphi}=P \theta\left(1-\eta \frac{E_{\mathrm{G}}}{h \nu}\right)\left[-\frac{\exp \left(-q_{\mathrm{T}} x\right)}{K^{(0)}}\right. \\
\left.+\frac{k}{\rho c L^{2}}\left[\frac{\exp \left(-q_{\mathrm{T}} x\right)}{p-\alpha}-\frac{\exp \left(-\sqrt{\left.\frac{p^{\prime}}{D} x\right)}\right.}{p^{\prime}-\alpha^{\prime}}\right]\right] \\
\text { avec } \quad \alpha=\frac{D_{\mathrm{T}}^{(0)}}{L^{2}} ; \quad p^{\prime}=p+\frac{1}{\tau} \text { et } \quad \alpha^{\prime}=\frac{1}{\tau} \\
\text { et } \quad k=\left(\eta E_{\mathrm{G}} / h v\right) / 1-\eta E_{\mathrm{G}} / h \nu .
\end{gathered}
$$

L'original de la fonction $\bar{\varphi}$ peut alors se trouver sur les tables [4] et peut s'écrire à l'aide des paramètres sans dimensions :

$$
\begin{gathered}
\xi=\frac{x}{L} ; \quad u=\alpha t=\frac{D_{\mathrm{T}}^{(0)} t}{L^{2}} ; \quad u^{\prime}=\alpha^{\prime} t=\frac{t}{\tau} \\
\varphi=\frac{P \theta\left(1-\eta \frac{E_{\mathrm{G}}}{h \nu}\right)}{2 \rho c L^{2}}\left\{-\frac{\xi}{u \sqrt{\pi u}} \exp \left(-\frac{\xi^{2}}{4 u}\right)+k \exp (u-\xi)\left[\operatorname{erfc}\left(\frac{\xi}{2 \sqrt{u}}-\sqrt{u}\right)-\operatorname{erfc}\left(\frac{\xi}{2 \sqrt{u^{\prime}}}-\sqrt{u^{\prime}}\right)\right]\right. \\
\left.+k \exp (u+\xi)\left[\operatorname{erfc}\left(\frac{\xi}{2 \sqrt{u}}+\sqrt{u}\right)-\operatorname{erfc}\left(\frac{\xi}{2 \sqrt{\overline{u^{\prime}}}}+\sqrt{\overline{u^{\prime}}}\right)\right]\right\} .
\end{gathered}
$$

(1) Dans l'annexe qui suit l'article, nous donnons quelques considérations sur la densité des paires électron-trou. 
En intégrant par rapport à $x$, on obtient la température $T$ :

$$
\begin{gathered}
T=\frac{P \theta\left(1-\eta \frac{E_{\mathrm{G}}}{h \nu}\right)}{2 \rho c L}\left\{\frac{2}{\sqrt{\pi}} \frac{\exp \left(-\frac{\xi^{2}}{4 u}\right)}{\sqrt{u}}-k \exp (u-\xi)\left[\operatorname{erfc}\left(\frac{\xi}{2 \sqrt{u}}-\sqrt{u}\right)-\operatorname{erfc}\left(\frac{\xi}{2 \sqrt{\overline{u^{\prime}}}}-\sqrt{u^{\prime}}\right)\right]\right. \\
\left.+k \exp (u+\xi)\left[\operatorname{erfc}\left(\frac{\xi}{2 \sqrt{u}}+\sqrt{u}\right)-\operatorname{erfc}\left(\frac{\xi}{2 \sqrt{u^{\prime}}}+\sqrt{\overline{u^{\prime}}}\right)\right]\right\} .
\end{gathered}
$$

Cette expression (14) peut se simplifier en tenant compte de ce que :

$$
\operatorname{erfc}(-\infty)=2 \text { et } \operatorname{erfc}(+\infty)=0\left(^{2}\right)
$$

car $u^{\prime}$ est très grand par rapport à $u$. On peut donc écrire :

$$
\begin{aligned}
T=\frac{P \theta}{2 \rho c L}\left(1-\eta \frac{E_{\mathrm{G}}}{h \nu}\right)\left\{\frac{2}{\sqrt{ } \pi} \cdot \frac{\exp \left(-\xi^{2} / 4 u\right)}{\sqrt{u}}\right. \\
+k \exp (u-\xi) \operatorname{erfc}\left(\sqrt{u}-\frac{\xi}{2 \sqrt{u}}\right) \\
\left.+k \exp (u+\xi) \operatorname{erfc}\left(\sqrt{u}+\frac{\xi}{2 \sqrt{u}}\right)\right\} \cdot \quad
\end{aligned}
$$

Cette équation peut s'écrire sous la forme :

$$
T=\frac{P \theta}{2 \rho c L}\left(1-\eta \frac{E_{\mathrm{G}}}{h \nu}\right)\left(\mathscr{T}_{1}+k \mathscr{T}_{\mathbf{2}}\right)
$$

où $\mathscr{T}_{1}$ est la fonction habituelle qui décrit la variation de la température en fonction de $t$ le long de l'échantillon [5], et $\mathscr{T}_{2}$ une fonction qui décrit la participation de l'effet photothermique à cette même variation. La fonction $\mathscr{T}_{1}$ admet un maximum pour $u=\xi^{2} / 2$, c'est-à-dire pour $t_{\mathrm{m}}^{(0)}=\frac{x^{2}}{2 D_{\mathrm{T}}^{(0)}}$. La détermination expérimentale de $t_{\mathrm{m}}^{(0)}$ permet de trouver le coefficient de diffusivité thermique du matériau étudié [6], [7].

Comme dans le cas de l'impulsion purement thermique, on définit, dans le cas présent, le coefficient de diffusivité thermique apparent par la relation :

$$
D_{\mathrm{T}}=\frac{x^{2}}{2 t_{\mathrm{m}}}
$$

où $t_{\mathrm{m}}$ sera le temps nécessaire pour que la température en un point $x$ d'un échantillon soumis à une impulsion lumineuse atteigne son maximum.

On peut alors définir la variation relative $\lambda$ par :

$\lambda=\frac{D_{\mathrm{T}}-D_{\mathrm{T}}^{(0)}}{D_{\mathrm{T}}^{(0)}}=\frac{K-K^{(0)}}{K^{(0)}}=-\frac{t_{\mathrm{m}}-t_{\mathrm{m}}^{(0)}}{t_{\mathrm{m}}^{(0)}}$

analogue à la variation $\frac{\sigma-\sigma^{(0)}}{\sigma^{(0)}}$ de la conductibilité

${ }^{(2)}$ On rappelle que :

avec

$$
\operatorname{erfc}(X)=1-\operatorname{erf}(X)
$$

$$
\operatorname{erf}(X)=-\operatorname{erf}(X)=\frac{2}{\sqrt{\pi}} \int_{0}^{x} \exp \left(-x^{2}\right) \mathrm{d} x
$$

électrique dans le phénomène bien connu de la photoconduction. (L'indice 0 repère les propriétés d'un semi-conducteur non éclairé.) On néglige évidemment la variation de la chaleur massique due à l'excès de la densité des porteurs, étant donné que la contribution de ceux-ci à la chaleur spécifique est négligeable pour un semi-conducteur à température ordinaire.

Dans la figure 1 , nous donnons les variations de $\mathscr{T}_{1}^{*}$

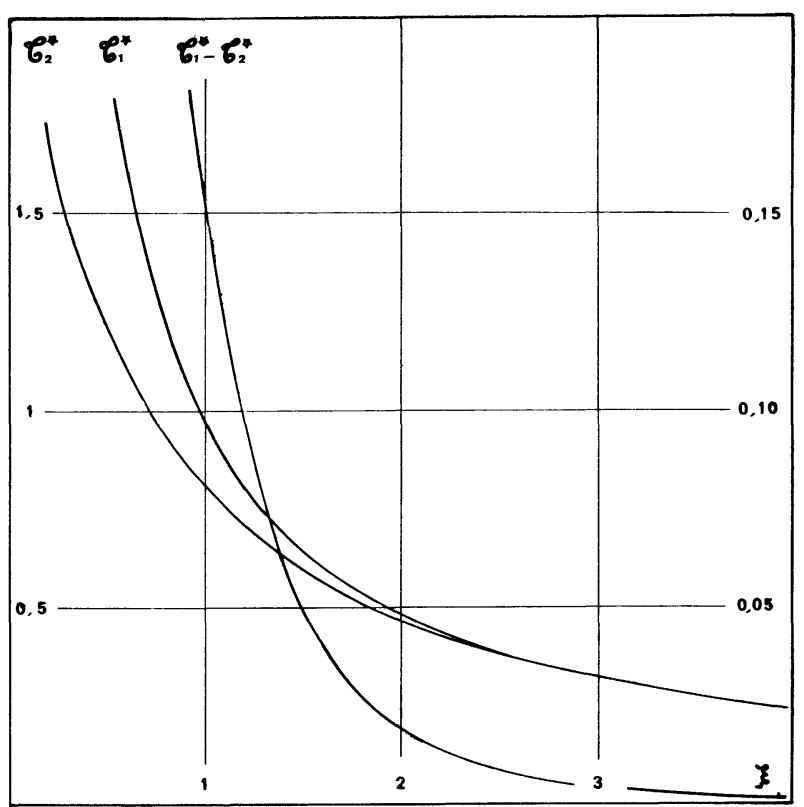

FIG. 1.

$\mathscr{T}_{1}^{*}=\mathscr{T}_{1}\left(\frac{\xi^{2}}{2}, \xi\right)$ où $\mathscr{T}_{1}$ est la fonction d'évolution thermique.

$\mathscr{T}_{2}^{*}=\mathscr{T}_{2}\left(\frac{\xi_{2}^{2}}{2}, \xi\right)$ où $\mathscr{T}_{2}$ est la fonction d'évolution photothermique.

et $\mathscr{T}_{2}^{*}$ et de $\mathscr{T}_{1}^{*}-\mathscr{T}_{2}^{*}$ en fonction de $\xi$ pour $u=\frac{\xi^{2}}{2}$. Nous voyons que, pour $\xi=4$ ou $x=4 L$, les fonctions $\mathscr{T}_{1}^{*}$ et $\mathscr{T}_{2}^{*}$ sont confondues, c'est-à-dire que les paires électron-trou se sont pratiquement entièrement recombinées.

Dans la figure 2 , nous donnons les variations de $\mathscr{T}_{1}$ et $\mathscr{T}_{2}$ en fonction de $u$ pour $\xi=1$.

Il est primordial de pouvoir calculer la variation $\lambda$. 


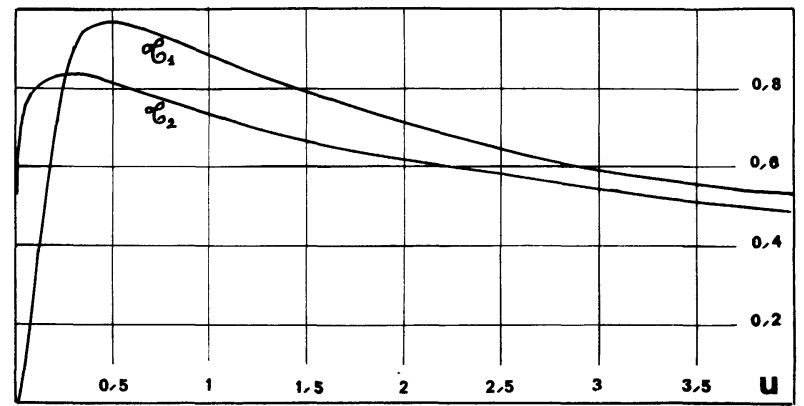

FIG. 2. - Variation de $\mathscr{T}_{1}$ et $\mathscr{T}_{2}$ en fonction de $u$.

Il est peu probable, étant donné la relative complexité de l'équation (15), de trouver une expression analytique simple exacte de $\lambda$. En développant la fonction (15) en série de Taylor autour de $u=\frac{\xi^{2}}{2}$, c'est-à-dire autour du maximum de $\mathscr{T}_{1}, \lambda$ peut s'exprimer sous la forme très simple :

$$
\lambda=\frac{2 k \xi^{2}\left(\mathscr{T}_{1}^{*}-\mathscr{T}_{2}^{*}\right)}{2 \mathscr{T}_{1}^{*}+k\left(\mathscr{T}_{1}^{*}-\mathscr{T}_{2}^{*}\right) \xi^{4}}
$$

qui peut s'écrire en tenant compte de ce que $\lambda$ est assez petit :

$$
\lambda=k \xi^{2} \frac{\mathscr{T}_{1}^{*}-\mathscr{T}_{2}^{*}}{\mathscr{T}_{1}^{*}} .
$$

L'expression (20) montre que $\lambda$, tout au moins en première approximation, est directement liée à $k$. Le facteur de proportionnalité dépend uniquement de $\xi$. On peut remarquer néanmoins sur la figure 3 que

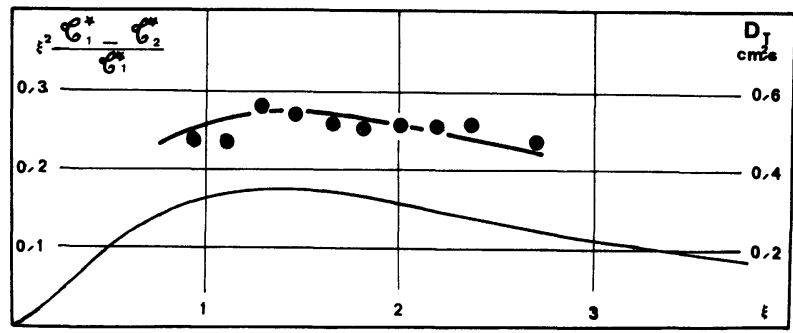

FIG. 3.

dans l'intervalle $1<\xi<2, \lambda$ varie peu et on pourrait prendre comme valeur, si la mesure expérimentale de $\lambda$ n'est pas très précise :

$$
\lambda=0,165 k \text { ou } k=6 \lambda \text { à } 5 \% \text { près. }
$$

L'effet photothermique pourrait donc permettre de déterminer, de manière très simple, par la mesure de $\lambda$, le facteur $k$ directement lié à la création des paires électron-trou et d'étudier par conséquent un problème fondamental dans les (S.C.). D'autre part, l'effet photothermique pourrait permettre de déterminer directement, par la mesure de la position $x_{\mathrm{m}}$ pour laquelle la diffusivité thermique apparente est maximum, la longueur de diffusion des paires électron- trou. La figure 3 montre en effet que $\lambda$ est maximum pour $\xi=1,35$. La longueur de diffusion $L$ sera alors simplement donnée par :

$$
L=0,74 x_{\mathrm{m}} .
$$

Comme on devait s'y attendre et comme les autres chercheurs ayant observé l'effet photothermique l'ont noté, l'équation (20) montre que, sous éclairement, la conductibilité thermique d'un semi-conducteur augmente.

La définition assez arbitraire de la diffusivité ther-

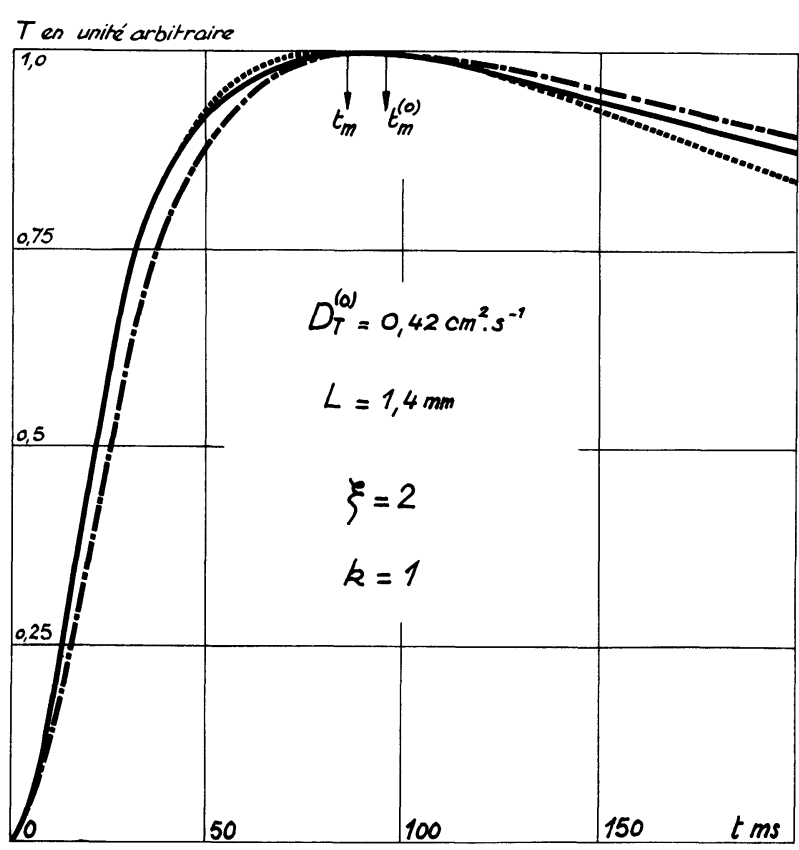

FIG. 4.

mique apparente que nous avons donnée précédemment (17) est assez bien justifiée par la figure 4. La courbe en trait plein donne la variation de la température calculée à partir de la formule (15) pour $\xi=2$ et $k=1$ avec $D_{\mathrm{T}}^{(0)}=0,42 \mathrm{~cm}^{2} / \mathrm{s}$ et $L=1,4 \mathrm{~mm}$.

La courbe en pointillé donne la variation de la température calculée en prenant une diffusivité thermique $D_{\mathrm{T}}$, tirée de la formule (21), et en remplaçant l'équation (2) par l'équation classique de la chaleur :

$$
\frac{\partial T}{\partial t}-D_{\mathrm{T}} \frac{\partial^{2} T}{\partial x^{2}}=0
$$

En trait discontinu, nous avons représenté la variation de $\mathscr{T}_{1}$. Les courbes sont ramenées à la même valeur maximale. On note le déplacement vers la gauche du maximum dû à l'effet photothermique.

III. Vérification expérimentale. - Pour vérifier nos hypothèses, nos résultats et nos conclusions, nous avons monté le dispositif expérimental suivant :

Deux plaquettes $\mathrm{A}$ et $\mathrm{B}$ de germanium $(30 \mathrm{~mm} \times$ $10 \mathrm{~mm} \times 2 \mathrm{~mm}$ ), taillées dans le même monocristal, 
sont disposées côte à côte et tenues au bout d'une vis micrométrique permettant de mesurer des déplacements au 1/100 de $\mathrm{mm}$ près. Le monocristal de germanium utilisé est de type $n$, dopé à l'antimoine, de résistivité $20 \Omega . \mathrm{cm}$, la durée de vie des porteurs minoritaires étant de $440 \mu$ s. La longueur de diffusion $L$ est donc voisine de $1,4 \mathrm{~mm}$.

Deux sondes s'appliquant respectivement sur les échantillons A et B permettent de suivre la variation de la température au point d'application en utilisant le pouvoir thermoélectrique élevé du germanium $\left(\sim 500 \mu \mathrm{V}\right.$.degré $\left.{ }^{-1}\right)$.

Tauc [8] a montré que le pouvoir thermoélectrique pouvait varier sous l'influence d'une modification de la densité des porteurs et il semblerait que la détermination de la température par l'effet Seebeck soit sujette à caution. Néanmoins, l'onde de concentration des porteurs arrive au point considéré beaucoup plus vite que l'onde thermique (voir annexe). La variation de la température est directement photographiée par l'intermédiaire d'une caméra équipée de polaroïd sur un oscillographe Tektronix, type 502 A. L'échantillon A est poli aussi bien que possible : polissage mécanique à l'aide de poudres abrasives dont la finesse est de plus en plus grande, polissage chimique au CP4 avec brome puis au CP4 sans brome. L'échantillon B par contre est sablé et la surface qui reçoit le rayonnement est revêtue d'une couche de peinture métallique d'aluminium afin d'éviter l'effet photothermique. Les deux échantillons reçoivent une même impulsion lumineuse délivrée par une lampe à xénon. Ils sont placés dans une enceinte susceptible d'être

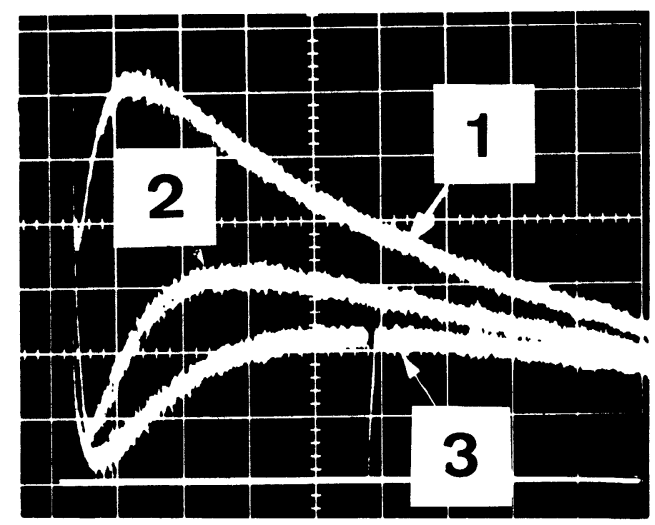

FIG. 5.

Gain : 0,1 $\mathrm{mV} /$ carreau; Vitesse : $20 \mathrm{~ms} / \mathrm{cm}$. $1: x=1,55 \mathrm{~mm} ; 2: x=2,55 \mathrm{~mm} ; 3: x=3,55 \mathrm{~mm}$.

vidée et d'être refroidie à la température de l'azote liquide.

Un exemple des oscillogrammes relevés pour l'échantillon A pour des positions différentes de l'échantillon est donné sur la figure 5.

Les résultats des différentes mesures effectuées sont

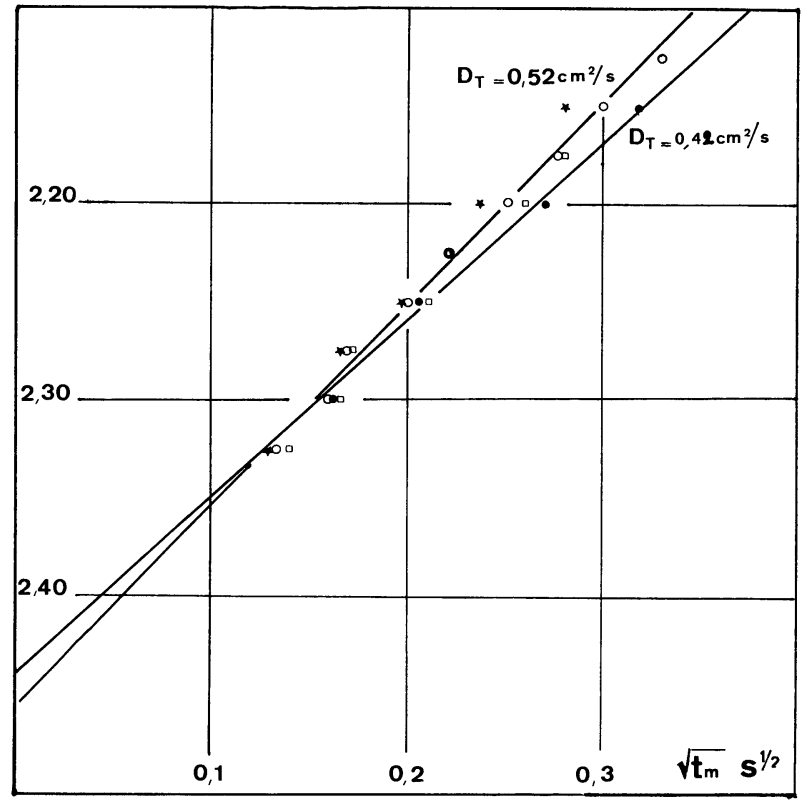

FIG. 6.

avec flash de $1600 \mathrm{~J}$
$\left.\begin{array}{l}1 \text { avec flash de } 800 \mathrm{~J} \\ \star \text { avec flash de } 400 \mathrm{~J}\end{array}\right\}$ échantillon poli
A échantillon non poli B

indiqués sur la figure 6. Dans cette figure, nous avons porté en ordonnées la position de la vis micrométrique. Chaque carreau correspond à un déplacement des échantillons de $1 \mathrm{~mm}$.

En abscisses, nous avons porté la racine carrée du temps nécessaire pour que la température en chaque point considéré soit maximum.

Pour l'échantillon $\mathrm{A}$, les mesures ont montré que la diffusivité thermique dépend peu de $P$ et de $\theta$, comme prévu théoriquement. Les variations observées peuvent être imputables soit à l'imprécision des mesures, soit encore à la variation de $\tau, L$ ou $\eta$ avec l'éclairement. Dans la figure 3 , nous avons reporté la variation de $D_{\mathrm{T}}$ en fonction de $\xi$ en attribuant à $L$ la valeur $1,4 \mathrm{~mm}$. L'allure de la variation de $D_{\mathrm{T}}$ semble, aux erreurs d'expérience près, assez semblable à la variation théorique de $\lambda$. La valeur moyenne des résultats obtenus pour la diffusivité thermique de l'échantillon A pour $1<\xi<2$ est :

$$
D_{\mathrm{T}}=0,52 \mathrm{~cm}^{2} / \mathrm{s} \pm 0,04 \mathrm{~cm}^{2} / \mathrm{s} \text {. }
$$

Quant à l'échantillon B, dont on peut dire que l'effet photothermique a été exclu par les traitements subis, on peut lui attribuer, en prenant la moyenne des résultats obtenus, une diffusivité thermique :

$$
D_{\mathrm{T}}^{(0)}=0,42 \mathrm{~cm}^{2} / \mathrm{s} \pm 0,03 \mathrm{~cm}^{2} / \mathrm{s} \text {. }
$$

Ce résultat est en bon accord avec les valeurs données dans la bibliographie : $0,35 \mathrm{~cm}^{2} / \mathrm{s}$ à $0,44 \mathrm{~cm}^{2} / \mathrm{s}$. 
La précision de nos mesures est assez faible, de l'ordre de $10 \%$, car $t_{\mathrm{m}}$ est difficile à déterminer exactement. En travaillant sur les valeurs moyennes obtenues pour les valeurs de $D_{\mathrm{T}}$ et $D_{\mathrm{T}}^{(0)}$ (ces valeurs moyennes étant calculées sur un très grand nombre de clichés réalisés), on peut attribuer à $\lambda$ la valeur 0,24 . En utilisant la relation (21), on trouve : $k=1,4$. On peut calculer $k$ en négligeant la vitesse de recombinaison superficielle des paires électron-trou, à l'aide de la formule :

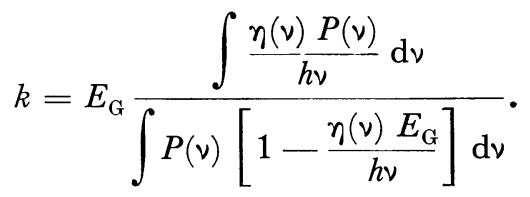

L'évaluation de $k$ est difficile dans le cas présent du fait de la complexité du spectre du flash xénon

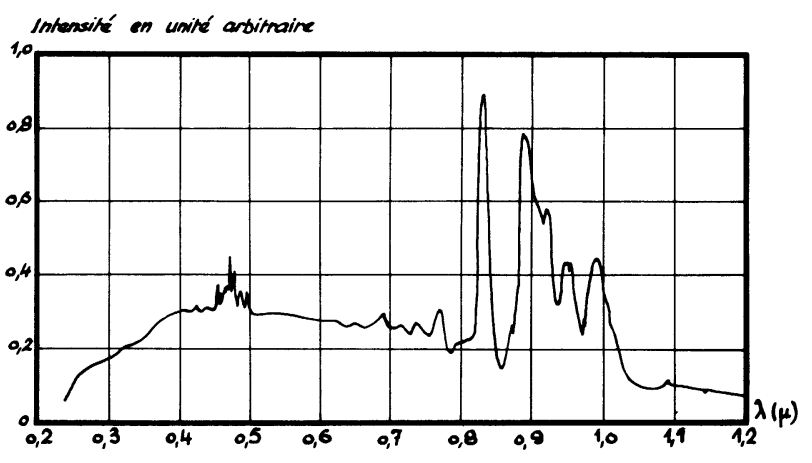

FIG. 7.

(fig. 7), de la transmission de la fenêtre utilisée, de la réflexion du germanium [9].

En supposant en première approximation que $P$ est constant dans un intervalle de longueur d'onde compris entre $1,2 \mu$ et $0,4 \mu$, que $\eta(\nu)$ est égal à 1 dans tout cet intervalle et en prenant $E_{\mathrm{G}}=0,8 \mathrm{eV}$, on trouve $k=1$. En tenant compte assez grossiè-

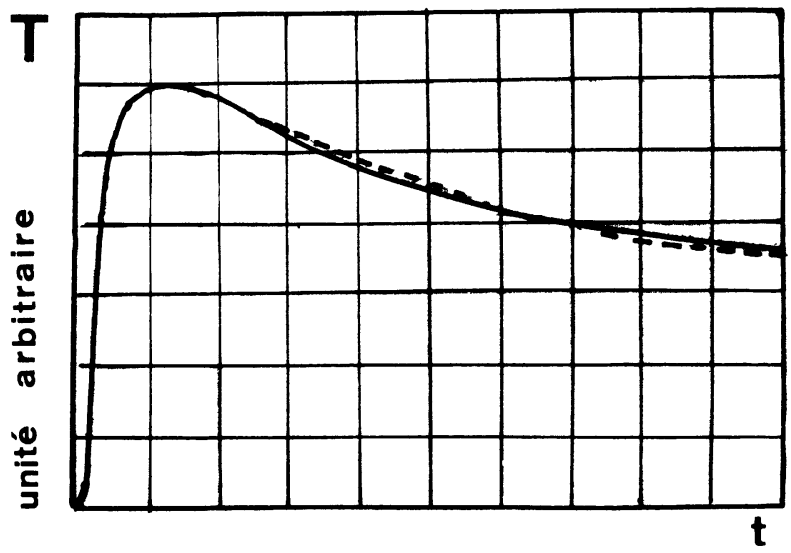

FIG. $8 .-\xi=1$ (un carreau $\rightarrow 20 \mathrm{~ms}$ )

courbe expérimentale.

-...- courbe théorique. rement des singularités du spectre du flash xénon et $\mathrm{du}$ pouvoir réflecteur du germanium, on trouve $k$ voisin de 1,2 , valeur assez peu différente de la valeur expérimentale trouvée.

Dans la figure 8, nous comparons une courbe expérimentale obtenue pour $\xi$ voisin de 1 avec la courbe théorique ramenée à la même valeur maximale en faisant $k=1,3$.

IV. Conclusions. - Malgré l'imprécision de nos résultats, signalée plus haut, nous pouvons conclure que la description simple de l'effet photothermique donnée ci-dessus est correcte, tout au moins en première approximation. Nous comptons reprendre les mêmes expériences en utilisant comme éclair une impulsion Laser dont le quantum d'énergie $h v$ est voisin de la largeur de bande interdite de l'échantillon semi-conducteur expérimenté, afin d'augmenter $\lambda$, ce qui permettrait de vérifier avec plus de précision les résultats de notre modèle.

Remerciements. - Je tiens particulièrement à remercier MM. les Professeurs M. Perrot et J. P. David qui ont dirigé ce travail et M. le Professeur Tavernier avec qui j'ai eu sur ce sujet de très bénéfiques discussions.

Manuscrit reçu le 5 décembre 1966.

\section{ANNEXE}

Détermination de la distribution des paires électrontrou dans l'échantillon. - L'équation (7) donne la transformée de Laplace de la densité des paires électron-trou. En passant à l'original, on trouve en introduisant les facteurs sans dimension $\xi$ et $u^{\prime}$ que

$$
\Delta p \sim \frac{1}{\sqrt{ } \pi} \frac{\exp \left(-\frac{\xi^{2}}{4 u^{\prime}}-u^{\prime}\right)}{\sqrt{u^{\prime}}}
$$

dont le maximum en fonction de $u^{\prime}$ est donné par

$$
u_{\mathrm{m}}^{\prime}=\frac{\sqrt{1+4 \xi^{2}}-1}{4} \text {. }
$$

Nous avons calculé aussi la distribution de la densité des porteurs en excès dans le cas où on ne néglige pas la vitesse de recombinaison superficielle sur la surface éclairée, et on trouve que :

$$
\begin{aligned}
& \Delta p \sim \frac{1}{\sqrt{\pi}} \frac{\exp \left(-\frac{\xi^{2}}{4 u^{\prime}}-u^{\prime}\right)}{\sqrt{u^{\prime}}} \\
& -a \exp \left[a \xi+\left(a^{2}-1\right) u^{\prime}\right] \operatorname{erfc}\left(\frac{\xi}{2 \sqrt{u^{\prime}}}+a \sqrt{u^{\prime}}\right)
\end{aligned}
$$

avec $a$, paramètre sans dimension défini par

$$
a=\mathrm{s} \sqrt{\frac{\tau}{D}}
$$


où $\mathrm{s}$ est la vitesse de recombinaison superficielle. Dans le cas où $a$ est de l'ordre de 1 (dans le cas de l'échantillon de germanium utilisé plus haut, cette condition correspond à $\mathrm{s}$ voisin de $350 \mathrm{~cm} / \mathrm{s}$ ), le second terme de la fonction (26) est petit devant le premier, ceci d'autant plus que $\xi$ est grand. Pour $\xi=2, u_{\mathrm{m}}^{\prime} \# 1,8$. Par suite, la densité des porteurs au point $x=2 L$ passe par un maximum au bout d'un temps de l'ordre de $0,8 \mathrm{~ms}$, temps considérablement plus court que le temps $t_{\mathrm{m}}$ (fig. 4) nécessaire pour que la température au même point passe par un maximum (90 ms mesuré expérimentalement).

\section{BIBLIOGRAPHIE}

[1] Gartiner (W. W.), Physical Review, 15 avril 1961, $112,2$.

[6] BURSUC (I.), Bulletin Scientifique Roumain, 1958, fasc. 1.

[2] Timberlake (A. B.), Davis (P. W.) et Shilitiday (T. S.), Advanced Energy Conversion, Pergamon Press, 1962, 2, 45-51.

[3] Weill et Leroux-Hugon, J. Physique Rad., décembre 1962, tome 23, suppl. au no 12, p. $215 \mathrm{~A}$.

[4] CARSLAW et JAEGER, Conduction of heat in solids, Clarendon Press.

Manuscrit reçu le 5 décembre 1966.

[5] Oualid (J.), J. Physique Rad., février 1961, 22, 124.

[7] Moya (G.), Thèse de Doctorat de $3^{\mathrm{e}}$ cycle de ChimiePhysique, Institut de Microcalorimétrie et de Thermogenèse. Faculté des Sciences de Marseille.

[8] TAUC (J.), Photo and thermoelectric effects in semiconductors, Pergamon Press, 1962.

[9] Koc, Czech. J. Phys., 1957, 7, 91. 